\title{
Fast Characterization of Mutually-Coupled Array Antennas Using Isolated Antenna Far-Field Data
}

\author{
Tomislav Marinović, Member, Dirk I. L. de Villiers, Senior Member, Dave J. Bekers, Senior Member, \\ Martin N. Johansson, Senior Member, Anders Stjernman, Rob Maaskant, Senior Member, \\ Guy A. E. Vandenbosch, IEEE Fellow
}

\begin{abstract}
A new method is proposed to analyze antenna arrays including mutual coupling, which is based on the concept of multiple scattering and relates to the iterative Jacobi and GaussSeidel methods. The method employs sampled far-field data of an isolated element, which can be obtained by any full-wave simulator and consists of far fields for excitation at the antenna feed and for plane waves with different angles of incidence. Mutual interactions between the array elements are modeled by approximating the incident field as a single dual-polarized plane wave taken from the spherical wave expansion of the scattered field from any other element in the array. The accuracy and run-time performances of the method are evaluated mainly by comparing simulations for several array geometries to MoMbased full-wave solutions. The method is primarily intended as a tool for the fast sequential analysis of arrays while varying the array lattice, particularly in case of irregular or sparse lattices and complex elements requiring dense meshes in fullwave simulators. The applications of the method may thus range from the systematic analysis of mutual coupling to optimization and synthesis.
\end{abstract}

Index Terms-Antenna arrays, mutual coupling, method of moments, multiple scattering, iterative methods, sparse arrays, radio astronomy

\section{INTRODUCTION}

Irregular sparse arrays (ISAs) provide several important benefits compared to regular antenna arrays. For instance, ISAs may have suppressed grating lobes due to their irregular lattice, and reduced coupling effects and enhanced frequency bandwidth due to a relatively large element spacing [1]. Additionally, if power and gain requirements allow, fewer elements can be used than in a regular array with the same

Submitted for review on Nov 8, 2019. Resubmitted on Apr 14, 2020. Resubmitted on May 30, 2020.

This project has received funding from the European Union's Horizon 2020 research and innovation program under the Marie Skłodowska-Curie grant agreement No. 721732.

T. Marinović is with KU Leuven, 3001 Leuven, Belgium (tomislav.marinovic@esat.kuleuven.be) and Chalmers University of Technology, 412 96 Gothenburg, Sweden (tommar@chalmers.se).

D. I. L. de Villiers is with Stellenbosch University, 7602 Stellenbosch, South Africa (ddv@sun.ac.za).

Dave J. Bekers is with TNO, the Netherlands organisation for applied scientific research, 2597 AK The Hague, The Netherlands (dave.bekers@tno.nl)

M. N. Johansson and A. Stjernman are with Ericsson Research, 41756 Gothenburg, Sweden (martin.n.johansson@ericsson.com, anders.stjernman@ericsson.com).

R. Maaskant is with Chalmers University of Technology, 41296 Gothenburg, Sweden (rob.maaskant@ @ chalmers.se) and Eindhoven University of Technology, 5612 AZ Eindhoven, The Netherlands (r.maaskant@tue.nl).

G. A. E. Vandenbosch is with KU Leuven, 3001 Leuven, Belgium (guy.vandenbosch@esat.kuleuven.be).

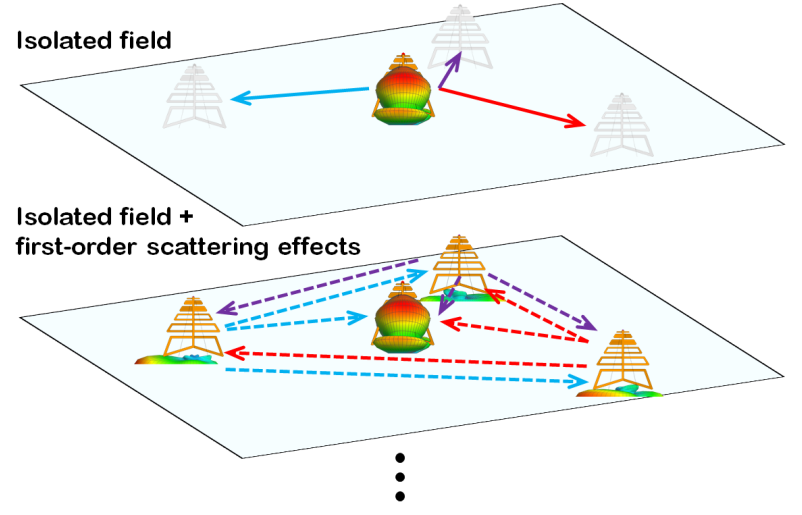

Isolated field + multiple-order scattering effects

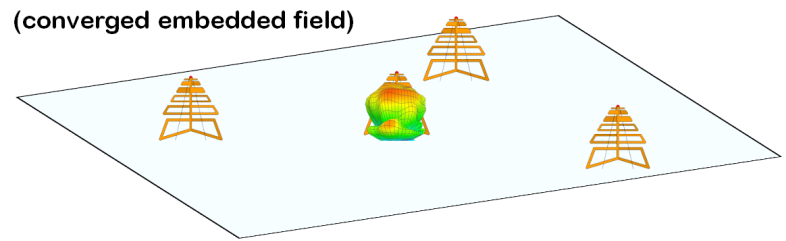

Fig. 1. Illustration of multiple scattering. Radiation from the excited element induces the scattered fields at all other elements. Each induced scattered field gives rise to a new set of scattered fields at all other elements. Iterating this interaction process results in a final field distribution given a predefined stopping criterion.

aperture. ISAs can be used in e.g. mobile communications [2], [3], satellite applications [4], and radio-astronomy [5].

Synthesizing large ISAs one often assumes idealized antenna elements with isolated or isotropic radiation patterns. This assumption may lead to suboptimal array configurations when the effects of mutual coupling (MC) cannot be ignored [6]. Furthermore, rapid evaluation of MC effects in large antenna arrays is particularly important in problems involving array optimization, where several (time-consuming) full-wave array simulations need to be performed [7]. Thus, efficient and accurate assessment of the impact of MC is important for the design and synthesis of ISAs [6]-[9].

MC can be seen as an aggregated effect of all mutual interactions between array elements which alters the radiation and impedance properties of an isolated element. The effect of MC is commonly observed through the embedded element pattern (EEP) and the array impedance or S-matrix. A review of general concepts and methods for the analysis of MC is presented in [10]. Small to mid-sized antenna arrays can be efficiently analyzed using full-wave methods, such as the 
method of moments (MoM) [11], [12]. However, the use of full-wave methods for the analysis of large array problems places a heavy burden on the computer memory and run time. Therefore, much attention has been committed to the development of efficient numerical techniques for large array modeling: the finite periodic structure approach [13], the multilevel fast multipole method (MLFMM) [14], [15], the domain Green's function method (DGFM) [16], and various methods based on the concept of synthesizing aggregate basis functions, such as macro basis functions (MBF) [17], synthetic functions [18], [19] and the characteristic basis functions (CBFM) [20], [21].

The use of direct methods for the analysis of electricallylarge complex structures or arrays can become prohibitively expensive with respect to CPU time and memory use. In that case iterative methods supplemented by the MLFMM can be a viable alternative [22, Ch. 8]. The MLFMM is mentioned in this respect as it cuts down substantially the complexity of the problem in each iteration, both in terms of memory use and run time $\left(\mathcal{O}\left(N_{\mathrm{x}} \log N_{\mathrm{x}}\right)\right.$ and $\mathcal{O}\left(N_{\mathrm{x}} \log ^{2} N_{\mathrm{x}}\right)$ instead of $\mathcal{O}\left(N_{\mathrm{x}}^{2}\right)$ and $\mathcal{O}\left(N_{\mathrm{x}}^{3}\right)$ in a conventional MoM, respectively, where $N_{\mathrm{x}}$ is the number of unknowns) [23]. The main drawback of iterative methods is that, depending on the problem at hand, many iterations may be required to get an accurate solution.

An important family of the methods to evaluate $\mathrm{MC}$ in antenna arrays is based on multiple scattering theory, where the effect of MC is seen as a collection of recurring elementary interactions between basic building blocks, as graphically illustrated in Fig. 1. Any modeling approach based on multiple scattering theory requires first the characterization of a basic building block or antenna-array element in isolation and, next, the description of the MC between different blocks or elements. The solution for the antenna array can then be obtained by using a direct or an iterative method. Various examples of using a direct method can be found in the literature, including the T-matrix method approach [24], the FEMbased generalized scattering matrix (GSM) approach [25], the canonical minimum scattering (CMS) antenna approach [26], the characteristic mode approach [27], linear embedding by Green's operators (LEGO) [28], and the expansion wave concept [29], [30]. In [24] and [30], the scattering response of the isolated element is obtained by illuminating the element with several plane waves impinging from different directions. The expansion waves between the array elements can be modeled using for example spherical waves [24], [25] or an analytical approximation of the Green's function for stratified dielectric media [30]. In all examples, the MC between different elements is accounted for by cascading the expansionwave responses of different elements, which involves matrix multiplication and inversion. The first examples of the modeling of multiple scattering using an iterative technique are found in [31]-[33]. In later years various dedicated alternatives for antenna arrays were developed, which can be classified as stationary (Jacobi and Gauss-Seidel) [34], [35] and Krylov subspace (GMRES) methods [36]. A comparison of these two types is presented in [37]. Additionally some authors show that iterative methods may benefit from MBF or CBFM formulations in the sense that convergence rate and stability are generally improved [38], [39].

The focus of this paper is on a novel iterative method for the analysis of antenna arrays including their MC, inspired by multiple scattering and related to the iterative Jacobi and Gauss-Seidel methods. Here, the isolated element is characterized by simulated full-wave far-field data. Such an approach allows using any present-day EM solver and avoids the need of specific analysis using e.g. circuit theory or Green's functions. Also, it allows for the analysis of antenna arrays of arbitrary size and shape with some arbitrary element. Similar to [30], the far-field data is obtained for excitation at the antenna feed and excitation by plane waves with different directions. However, the MC between different elements is modeled by approximating the incident (near-)field with a pair of plane waves with orthogonal polarization taken from the spherical wave expansion (SWE) of the incident field. Since these plane waves can be matched straightforwardly to the simulated element data, the interaction process is computationally inexpensive. As compared to the MoM/MLFMM, the run time of our method scales with the number of elements rather than the number of basis functions, since full-wave characterization is performed for the isolated element only. As such, our method is primarily intended as a faster alternative to iterative methods supplemented by the MLFMM. A first proof-ofconcept is presented in [40], which demonstrates the accurate approximation of the EEP of an array of two dipoles in freespace.

The paper is organized as follows. Sec. II provides a theoretical formulation of our method for arrays in free and half space and describes the propagation mechanism and input data. Next, Sec. III presents a numerical validation of the method by considering some medium-sized arrays and analyzing the accuracy of the method for both S-parameter and far-field estimation as compared to the MoM/MLFMM of the commercial solver FEKO. Both the array lattice (regular and irregular/sparse) and the type of element (dipole, planar inverted-F antenna (PIFA), and log-periodic) are varied in the numerical examples. Subsequently, an analysis of the computational complexity of the method is presented together with an overview of computational times obtained on a specific platform, which are compared to those recorded using FEKO. Finally, conclusions are drawn in Sec. IV.

\section{Theoretical Formulation}

\section{A. Derivation of the Iterative Method}

A classical MoM matrix system $\boldsymbol{Z} \boldsymbol{J}=\boldsymbol{V}$ relates discretized (surface) currents $\boldsymbol{J}$ to voltages $\boldsymbol{V}$. Applying the iterative Jacobi method to this equation to resolve $\boldsymbol{J}$ results in the iterative scheme $\boldsymbol{J}^{(q)}=\boldsymbol{Z}_{\text {on }}^{-1}\left(\boldsymbol{V}-\boldsymbol{Z}_{\text {off }} \boldsymbol{J}^{(q-1)}\right)$ for $q \geq 1$, where $\boldsymbol{J}^{(0)}=\boldsymbol{Z}_{\text {on }}^{-1} \boldsymbol{V}$ and $\boldsymbol{Z}=\boldsymbol{Z}_{\text {on }}+\boldsymbol{Z}_{\text {off }}$ with $\boldsymbol{Z}_{\text {on }}$ and $Z_{\text {off }}$ consisting of the diagonal and off-diagonal elements of $Z$, respectively. For an array of $N$ separate elements, one can also view $\boldsymbol{Z}$ as a block matrix with blocks $\boldsymbol{Z}_{m m}$ representing the intra-element coupling and blocks $\boldsymbol{Z}_{m m^{\prime}}\left(m \neq m^{\prime}\right)$ representing the inter-element coupling. In that case, $Z_{\text {on }}$ is a diagonal block matrix containing the blocks $\boldsymbol{Z}_{m m}$ on its diagonal and $\boldsymbol{Z}_{\text {off }}$ is an off-diagonal block matrix containing 
all blocks $\boldsymbol{Z}_{m m^{\prime}}$. Consequently, the iteration scheme can be written down per element current $\boldsymbol{J}_{m}$,

$$
\boldsymbol{Z}_{\mathrm{on}, m m} \boldsymbol{J}_{m}^{(q)}=\boldsymbol{V}_{m}-\sum_{m^{\prime}=1, m^{\prime} \neq m}^{N} \boldsymbol{Z}_{\mathrm{off}, m m^{\prime}} \boldsymbol{J}_{m^{\prime}}^{(q-1)},
$$

where the left and right-hand sides represent voltages. Such a scheme is for example described in [39, Eq. (4)].

Inspired by this iterative scheme, one can envisage a similar iterative scheme in terms of electric fields instead of voltages, which is described in [40] for EEPs. Here we introduce a more general description. Let $\underline{\boldsymbol{E}}_{m}^{(q)}$ be the electric field of element $m$ in iteration $q \geq 1$. Then,

$$
\underline{\boldsymbol{E}}_{m}^{(q)}=\underline{\boldsymbol{E}}_{m}^{(0)}+\sum_{\substack{m^{\prime}=1 \\ m^{\prime} \neq m}}^{N} \underline{\underline{\boldsymbol{E}}}_{m m^{\prime}}^{\mathrm{s}(0)} \underline{\boldsymbol{E}}_{m^{\prime}}^{(q-1)} .
$$

Here, $\underline{\boldsymbol{E}}_{m}^{(0)}$ is the field of element $m$ in isolation and $\underline{\underline{E}}_{m m^{\prime}}^{\mathrm{s}(0)}$ is a dyad which transforms the electric field of element $m^{\prime}$, generated by a source with unit amplitude, into the field scattered by element $m$.

By Schelkunoff's equivalence principle, the angular components of the field $\underline{\boldsymbol{E}}_{m^{\prime}}^{(q-1)}$ on a minimum sphere which encompasses all radiating parts of the element provide all information for the complete radiated or scattered field description. Note that other non-overlapping surfaces can be used, but a sphere allows a straightforward description.

Therefore, it is convenient to define local spherical coordinate systems at elements $m$ and $m^{\prime}$ with unit vectors $\{\hat{\boldsymbol{r}}, \hat{\boldsymbol{\theta}}, \hat{\boldsymbol{\phi}}\}$ and $\left\{\hat{\boldsymbol{r}}^{\prime}, \hat{\boldsymbol{\theta}}^{\prime}, \hat{\boldsymbol{\phi}}^{\prime}\right\}$. The field vector of element $m^{\prime}$ in (2) can then be restricted to the angular components on its minimum sphere, $\left.\underline{\boldsymbol{E}}_{m^{\prime}}^{(q-1)}\right|_{\theta^{\prime}, \phi^{\prime}}$, where $\left.\right|_{\theta^{\prime}, \phi^{\prime}}$ indicates the restriction. Additionally, the dyad $\underline{\underline{E}}_{m m^{\prime}}^{\mathrm{s}(0)}$ can be expressed as

$$
\underline{\underline{E}}_{m m^{\prime}}^{\mathrm{s}(0)}=\left[\begin{array}{ll}
E_{m m_{\theta, \theta^{\prime}}^{\mathrm{s}}}^{\mathrm{s}(0)} & E_{m m_{\theta, \phi^{\prime}}^{\prime}}^{\mathrm{s}(0)} \\
E_{m m_{\phi, \theta^{\prime}}^{\mathrm{s}(0)}}^{\mathrm{s}} & E_{m m_{\phi, \phi^{\prime}}^{\mathrm{s}}}^{\mathrm{s}(0)} \\
E_{m m_{r, \theta^{\prime}}^{\mathrm{s}}(0)}^{\prime} & E_{m m_{r, \phi^{\prime}}^{\mathrm{s}}(0)}^{\prime}
\end{array}\right],
$$

where the columns represent transforms that yield the spherical components of the fields at element $m$ due to $\hat{\boldsymbol{\theta}}^{\prime}$ and $\hat{\boldsymbol{\phi}}^{\prime}$ polarized fields originating from element $m^{\prime}$.

Instead of restricting the field $\underline{\boldsymbol{E}}_{m^{\prime}}^{(q-1)}$ of element $m^{\prime}$ to its own minimum sphere, we can first propagate the field and restrict it to its tangential components on the minimum sphere of element $m$. In notation, $\left.\underline{\boldsymbol{E}}_{m^{\prime}}^{(q-1)}\right|_{\theta^{\prime}, \phi^{\prime}}$ is replaced by $\left.\underline{\boldsymbol{E}}_{m^{\prime}}^{(q-1)}\right|_{\theta, \phi}=\underline{\boldsymbol{w}}_{m m^{\prime}}^{(q-1)}=\left[\begin{array}{ll}w_{m m^{\prime}, \theta}^{(q-1)} & w_{m m^{\prime}, \phi}^{(q-1)}\end{array}\right]^{T}$. Consequently, the primes in the angular components of (3) are removed. To elucidate the multiple-scattering process, Fig. 2 illustrates the first few iterations for two elements, where only one element has a voltage source.

Let us now consider an element $p$ whose field can be viewed as being composed of fields induced by elements $m$, each of which is described by (2) with $\underline{\boldsymbol{E}}_{m^{\prime}}^{(q-1)}$ replaced by $\underline{\boldsymbol{w}}_{m m^{\prime}}^{(q-1)}$ as described above. In the same notation, $\underline{\boldsymbol{E}}_{m}^{(q)}$ can be replaced by $\underline{\boldsymbol{w}}_{p m}^{(q)}$ by applying the restriction $\left.\right|_{\theta, \phi}$ at the minimum sphere

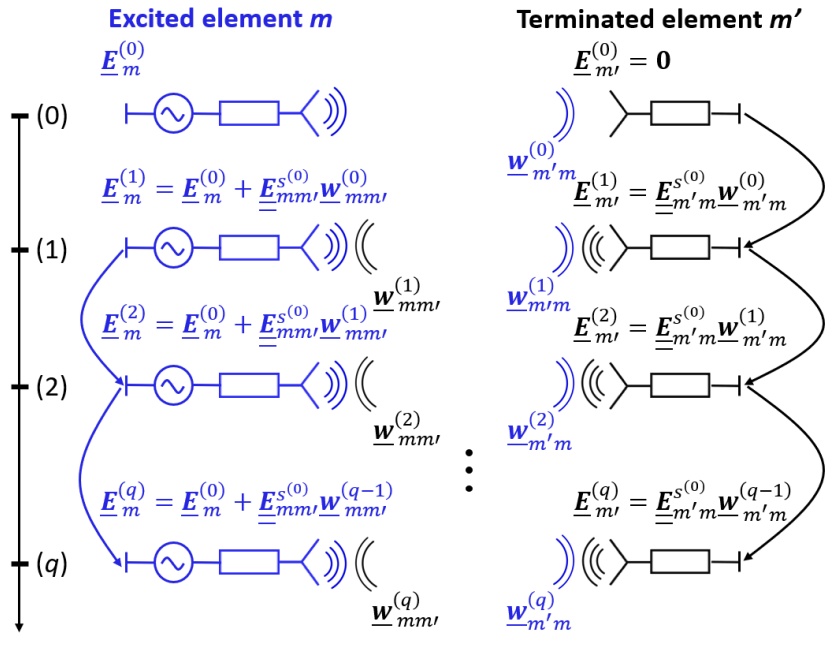

Fig. 2. Illustration of the iteration process as described by (2): radiation from the element $m$ (blue) excited by a voltage source illuminates the terminated element $m^{\prime}$ (black) inducing the scattered field and triggering a series of scattering interactions between the two elements.

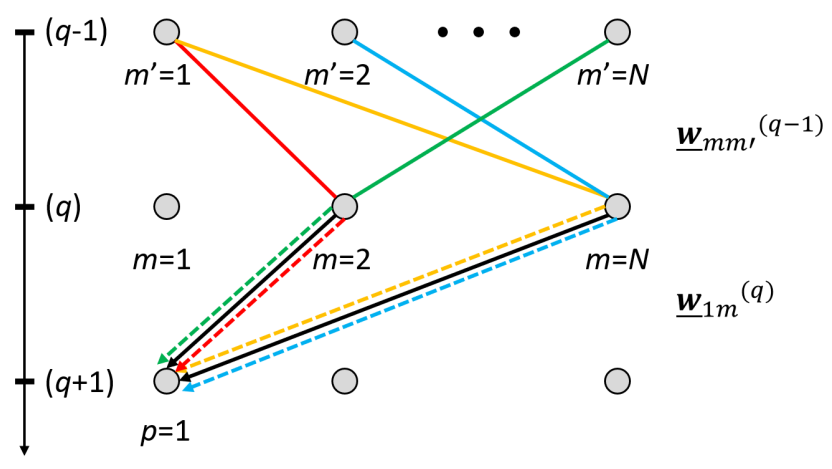

Fig. 3. A visual representation of (4) with respect to element $p=1$. Each field incident at this element, $\underline{\boldsymbol{w}}_{1 \mathrm{~m}}^{(q)}$, includes the isolated field radiated by element $m, \underline{\boldsymbol{E}}_{m}^{(0)}$ (solid black line), and all fields scattered by element $m$ (dashed colored lines) due to a set of incident fields computed in the previous iteration, $\underline{\boldsymbol{w}}_{m m^{\prime}}^{(q-1)}$ (solid colored lines).

of element $p$, where $\theta$ and $\phi$ relate to the coordinate system of element $p$. Consequently,

$$
\underline{\boldsymbol{w}}_{p m}^{(q)}=\left.\underline{\boldsymbol{E}}_{m}^{(0)}\right|_{\theta, \phi}+\left.\sum_{\substack{m^{\prime}=1 \\ m^{\prime} \neq m}}^{N}\left(\underline{\underline{E}}_{m m^{\prime}}^{\mathrm{s}(0)} \underline{\boldsymbol{w}}_{m m^{\prime}}^{(q-1)}\right)\right|_{\theta, \phi} .
$$

A visual representation of the updates of the "weights" $\underline{\boldsymbol{w}}_{p m}^{(q)}$ in this iteration process is given in Fig. 3.

In case the elements are farther apart, spheres larger than the minimum ones can be used as long as they remain disjoint. Approaching the far-field distance, the tangential fields on a sphere of the scattering element can be reduced to a single direction of incidence. In this way, the vector field $\underline{\boldsymbol{w}}_{m m^{\prime}}^{(q-1)}$ on the sphere turns into the vector $\underline{\check{\boldsymbol{w}}}_{m m^{\prime}}^{(q-1)} \in \mathbb{C}^{2 \times 1}$. Then, the far-field equivalent of (2) is

$$
\underline{\boldsymbol{F}}_{m}^{(q)}=\underline{\boldsymbol{F}}_{m}^{(0)}+\sum_{\substack{m^{\prime}=1 \\ m^{\prime} \neq m}}^{N}\left(\underline{\underline{\boldsymbol{F}}}_{m m^{\prime}}^{\mathrm{s}(0)} \underline{\check{\boldsymbol{w}}}_{m m^{\prime}}^{(q-1)}\right),
$$


where $\underline{\boldsymbol{F}}_{m}^{(0)}$ and $\underline{\underline{\boldsymbol{F}}}_{m m^{\prime}}^{\mathrm{s}(0)}$ are the far-field equivalents of $\underline{\boldsymbol{E}}_{m}^{(0)}$ and the dyad in (3), respectively, where the dyad reduces to a $2 \times 2$ transformation. The total far-field is obtained by linear superposition of element far-field patterns. At iteration $q$ this field is given by

$$
\underline{\boldsymbol{F}}^{(q)}=\sum_{m=1}^{N} \underline{\boldsymbol{F}}_{m}^{(q)} \mathrm{e}^{j \boldsymbol{k} \cdot \boldsymbol{r}_{m}},
$$

where $\boldsymbol{k}=k(\sin \theta \cos \phi \hat{\boldsymbol{x}}+\sin \theta \sin \phi \hat{\boldsymbol{y}}+\cos \theta \hat{\boldsymbol{z}})$ and $\boldsymbol{r}_{m}$ is the reference point of element $m$. In the cases of singleelement and full-array excitation, (6) corresponds to the EEP and the array pattern, respectively.

The currents at the antenna terminals can be expressed similarly. For the far-field case in (5), the expression is

$$
I_{m}^{(q)}=I_{m}^{(0)}+\sum_{\substack{m^{\prime}=1 \\ m^{\prime} \neq m}}^{N} \boldsymbol{I}_{m m^{\prime}}^{\mathrm{i}(0)} \underline{\check{\boldsymbol{w}}}_{m m^{\prime}}^{(q-1)},
$$

where $I_{m}^{(0)}$ is the current at the terminals of the isolated element and $\boldsymbol{I}_{m m^{\prime}}^{\mathrm{i}(0)}=\left[\begin{array}{ll}I_{m m_{\theta}^{\prime}}^{\mathrm{i}(0)} & I_{m m_{\phi}^{\prime}}^{\mathrm{i}(0)}\end{array}\right] \in \mathbb{C}^{1 \times 2}$ represents the induced currents at the terminals of element $m$ due to unit-amplitude $\hat{\boldsymbol{\theta}}$ and $\hat{\boldsymbol{\phi}}$-polarized plane waves impinging from direction $\boldsymbol{r}_{m m^{\prime}}=\boldsymbol{r}_{m}-\boldsymbol{r}_{m^{\prime}}$, i.e., the offset between the reference points $\boldsymbol{r}_{m}$ and $\boldsymbol{r}_{m^{\prime}}$ of the two elements. When the currents at all terminals are known, S-parameters can be extracted using standard relations [41].

One key step remains to be clarified: the propagation of the fields from one element to another. Iteration $q=1$ requires the evaluation of $\left.\underline{\boldsymbol{E}}_{m}^{(0)}\right|_{\theta, \phi}$ used in (4), or its far-field equivalent from (5), which means first evaluating the field of the isolated element at the minimum sphere of element $p$ and, next, restricting to its angular components in the coordinate system of element $p$. Similarly, iteration $q \geq 2$ requires the evaluation of $\left.\left(\underline{\underline{\boldsymbol{E}}}_{m m^{\prime}}^{\mathrm{s}(0)} \underline{\boldsymbol{w}}_{m m^{\prime}}^{(q-1)}\right)\right|_{\theta, \phi}$, which comes down to similar steps and, additionally, evaluating the scattered field of element $p$.

For the first step, the most versatile and computationally efficient way seems to expand the field of a single isolated element into some finite set of analytically known modes. Examples of modal representations include the SWE [42, Ch. 3.10] or the plane-wave spectrum (PWS) approach. In principle, both SWE and PWS allow for the modeling of the evanescent field effects, but the accuracy of the expansion at some point in space depends on the number of modes and the information used to determine the expansion coefficients. If these coefficients are determined from sampled field-data (with sufficient density) at a sphere slightly bigger than the minimum sphere which encompasses all radiating parts of the isolated element, the expansion will provide reasonably accurate field values at any point in the near field outside the chosen sphere [43]. However, if the field is sampled at a sphere with an electrically larger radius, or far-field data is employed, the expansion will not describe all near-field effects, since the data do not exhibit the evanescent field information in full. For dipoles with different lengths and SWEs with different numbers of modes, we observed that the SWE can still accurately describe the field components at points close to the minimum sphere (errors in the order of a few percent), when the expansion coefficients are determined from far-field data, although the classical far-field conditions are not all met (specifically, distance not being much larger than wavelength and effective aperture of the antenna).

For the second and third steps of restricting the field to the $\theta, \phi$-components at element $p$ and generating the scattered field of element $p$, the most straightforward approach is as follows. Turn first the field expansion of element $m$ into a single plane wave at element $p$. Next, estimate the resulting scattered field of element $p$ for that (incident) plane wave from simulated scattered fields of the isolated element for plane waves with different directions of incidence and unit amplitude. This approach has been investigated in [40], where the field expansion is SWE (and therefore the approach is termed "SW-PW"). Directivity simulations for two dipoles show that this approach has similar error behaviour as function of dipole length and spacing as the more rigorous approach (termed "SW") of determining the scattered field at element $p$ directly from the SWE of element $m$, which is more computationally intensive. Both approaches outperform largely the less rigorous approach (termed "PW") of using only a single plane wave in the field expansion of element $m$ instead of multiple spherical waves. It should be noted that the "SW-PW" approach fits directly in our description above of the iterations process and keeps the computational cost per iteration at a minimum.

The single plane wave is obtained from the SWE as follows. In case elements are in each other's far fields, the effect of MC is described by a pair of plane waves with orthogonal polarization, traveling along the direct paths between the reference points of each pair of elements. Here, the antenna reference point (origin of the local coordinate system) can be chosen rather arbitrarily, e.g., in the feed point. The SWE-based field originating from element $m^{\prime}$ is evaluated in the reference point of element $m$, denoted as $\underline{\boldsymbol{E}}_{m^{\prime}}^{\mathrm{SWE}}\left(\boldsymbol{r}_{m m^{\prime}}\right)$. Alternatively, we may consider the PWS in the same point expressed as [44]

$$
\begin{aligned}
& \underline{\boldsymbol{E}}_{m^{\prime}}^{\mathrm{PWS}}\left(\boldsymbol{r}_{m m^{\prime}}\right)=\frac{1}{2 \pi} \int_{-\infty}^{\infty} \int_{-\infty}^{\infty} \underline{\boldsymbol{T}}_{m^{\prime}}\left(k_{x}, k_{y}\right) . \\
& e^{j\left(k_{x} x_{m m^{\prime}}+k_{y} y_{m m^{\prime}}\right)} e^{j k_{z} z_{m m^{\prime}}} d k_{x} d k_{y},
\end{aligned}
$$

where $k_{x}, k_{y}$ and $k_{z}=\sqrt{k-k_{x}^{2}-k_{y}^{2}}$ are the Cartesian components of the wave vector $\boldsymbol{k}=k_{x} \hat{\boldsymbol{x}}+k_{y} \hat{\boldsymbol{y}}+k_{z} \hat{\boldsymbol{z}}$, with $\hat{\boldsymbol{z}}=$ $\hat{\boldsymbol{r}}_{m m^{\prime}}$. Here, $k_{x}=k \sin \alpha \cos \beta, k_{y}=k \sin \alpha \sin \beta, k_{z}=$ $k \cos \alpha$, with $\alpha=\arcsin \left(\sqrt{\left(k_{x}^{2}+k_{y}^{2}\right) / k^{2}}\right)$ and $\beta=$ $\arctan \left(k_{y} / k_{x}\right)$. Moreover, $\underline{\boldsymbol{T}}_{m^{\prime}}\left(k_{x}, k_{y}\right)$ is the plane wave spectrum at $z_{m^{\prime}}$, while $\underline{\boldsymbol{T}}_{m^{\prime}}\left(k_{x}, k_{y}\right) e^{j k_{z} z_{m m^{\prime}}}$ is the plane spectrum at $z_{m m^{\prime}}$, as detailed in [44]. With $r_{m m^{\prime}} \rightarrow \infty$, the integral in Eq. (8) is dominated by a spectral component in the direction $\hat{\boldsymbol{r}}_{m m^{\prime}}$, i.e., $\left\{0,0, k_{z}\right\}$. Spectral components in non-radial directions exhibit faster phase term oscillations and, hence, their contribution to the final solution can be ignored [45, p. 99]. The single plane wave may assume any two polarization directions which are mutually orthogonal and, of course, orthogonal to the direction of propagation. A suitable selection is a pair of polarization vectors corresponding to $\hat{\boldsymbol{\theta}}$ and $\hat{\boldsymbol{\phi}}$. Then, a straightforward approach to determine the complex magnitudes of the vector components of the plane wave is to project the SWE-based $\hat{\boldsymbol{\theta}}^{\prime}$ and $\hat{\boldsymbol{\phi}}^{\prime}$ field components 
of element $m^{\prime}$ onto the spherical vectors $\hat{\boldsymbol{\theta}}$ and $\hat{\boldsymbol{\phi}}$ at the reference point $\boldsymbol{r}_{m}$ of element $m$.

In case elements are not in each other's far fields, a similar procedure can be followed, but the choice of the antenna reference point needs attention (a.o. because the relation between distance and phase is not necessarily linear). For the simulated antennas in this paper, the element's reference point is positioned in the center of the minimum sphere. A rigorous approach is to define the reference point as the unique radiation center obtained by minimizing a $L_{2}$ measure for the modal content [46]. Finally, notice that, due to the SW-PW transformation, the vector fields in (4) are replaced by vectors in $\mathbb{C}^{2 \times 1}$, as in (5) and (7), but the whole procedure is not a pure far-field or PW transformation because of the SWE being evaluated at element separation distance.

In this paper, all elements in the array are assumed to be the same and have thus the same isolated fields translated to their reference points. Moreover, simulated far-field data of the isolated element are used to compose SWEs of the fields for different excitations.

These data are detailed as follows:

- Element excited by a voltage source of $1 \mathrm{~V}$

i. Radiated far-field

ii. Current at the antenna terminals

- Terminated element excited by $\hat{\boldsymbol{\theta}}^{\prime}$ and $\hat{\boldsymbol{\phi}}^{\prime}$-polarized plane waves of $1 \mathrm{~V} / \mathrm{m}$ incident from different directions

i. A set of scattered far-fields

ii. A set of induced currents at the antenna terminals.

These data can be readily obtained by any available EMfield solver and need to be computed only once. Note that the SWE allows to get the scattered far-field pattern of the (isolated) element for any direction of incidence instead of only the directions in the full-wave simulated data. First the expansion coefficients of the SWEs of the simulated fields are determined by taking classical inner products $\left(L_{2}\right.$ on a unit sphere with numerical integration) of the orthogonal SWE modes and the field data. Next, these expansion coefficients are interpolated to get the expansion coefficients of the SWEs for non-simulated directions. Such an interpolation is more robust than interpolating the field data directly, especially in case of coarse sampling of incidence angles. For completeness, note that the effects of MC modeled in this paper include radiated waves only. Inclusion of surface wave effects in dielectric substrates is not part of this study.

\section{B. Infinite ground-plane model}

The effect of an infinite ground plane is modeled using image theory. Two approaches can be distinguished depending on the type of antenna geometry. Full-wave characterization of an antenna whose basic geometry does not comprise the ground plane, e.g. a dipole or a horn antenna, is performed without the ground plane. When such a structure is mounted above an infinite ground plane, its virtual mirror-image is created below the ground plane such that

$$
\begin{aligned}
& \underline{\boldsymbol{F}}_{\theta}^{\prime}(\theta, \phi)=\underline{\boldsymbol{F}}_{\theta}(\pi-\theta, \phi) \\
& \underline{\boldsymbol{F}}_{\phi}^{\prime}(\theta, \phi)=-\underline{\boldsymbol{F}}_{\phi}(\pi-\theta, \phi) .
\end{aligned}
$$

In this way, the number of array elements is effectively doubled prior to using the iterative method (4). Full-wave characterization of antennas whose basic geometry comprises the ground plane, e.g. metallic patches or PIFAs, needs to be performed in the presence of the ground plane. The exported far-field results contain only the hemispherical data corresponding to $0 \leq \theta \leq \pi / 2$. Accurate $\mathrm{SWE}$ of such discontinuous data would require an infinite number of SWE modes. To reduce the mode content, image data corresponding to $\pi / 2<\theta \leq \pi$ is first created using (9a) and (9b) to enforce continuity before the resulting field is expanded. In this model, each $\underline{\boldsymbol{w}}_{p m}^{(q)}$ needs to be scaled by a factor of $1 / 2$ to compensate for the doubling of the total energy contained in the far-field.

\section{Numerical ANALysis AND VALidation}

In this section, a numerical analysis of the proposed method is presented. First, general aspects of convergence are discussed in relation to the classical iterative methods and a measure of error is introduced for the iteration scheme. Next, the simulation settings are described followed by numerical results for different types of arrays, comparison with fullwave simulation, and numerical analysis of convergence and parameter dependence. Finally, complexity and run time are analyzed.

\section{A. Convergence considerations}

In case the classical iterative Jacobi method is applied to a MoM matrix system, as in (1), its convergence can be analyzed relatively straightforwardly. A sufficient convergence condition is $\left\|\boldsymbol{Z}_{\text {on }}^{-1} \boldsymbol{Z}_{\text {off }}\right\|<1$ for some norm, while a necessary and sufficient convergence condition is $\rho\left(\boldsymbol{Z}_{\text {on }}^{-1} \boldsymbol{Z}_{\text {off }}\right)<1$ with $\rho(\cdot)$ the spectral radius [47, Theorems 8.9-8.10]. The Jacobi method will also converge if $\boldsymbol{Z}$ is strictly or irreducibly diagonally dominant [48, Theorem 3.10]. Consequently, stronger levels of MC in closely spaced antenna arrays may lead to convergence issues in the iterative Jacobi method. An example of the iterative Jacobi method applied to an antenna-array MoM system is described in [39].

The convergence of our iterative method, inspired by the multiple scattering in an antenna array and the availability of (far-)field data of an isolated element, is harder to assess from a theoretical point-of-view. In the propagation step, a finite SWE is used which is collapsed at each element to a pair of plane waves with orthogonal polarization. The corresponding element scattered fields are then obtained from the simulations of an isolated element for plane-wave incidence by some fullwave simulator with its own numerical specifications in terms of meshing, coupling computation, and inversion. Hence, the theoretical analysis of convergence does not seem obvious. An alternative is to analyze convergence numerically via the relative difference of aggregate weight vectors in subsequent iterations. The aggregate weight vector $\boldsymbol{W}^{(q)} \in \mathbb{C}^{2 N^{2}}$ is a vector with the values of $\check{\boldsymbol{w}}_{p m, \theta}^{(q)}$ and $\underline{\underline{\boldsymbol{w}}}_{p m, \phi}^{(q)}$ for all $p, m$, and the relative difference is defined by

$$
\rho^{(q)}=\frac{\left\|\boldsymbol{W}^{(q)}-\boldsymbol{W}^{(q-1)}\right\|_{2}}{\left\|\boldsymbol{W}^{(q)}\right\|_{2}}<\delta .
$$




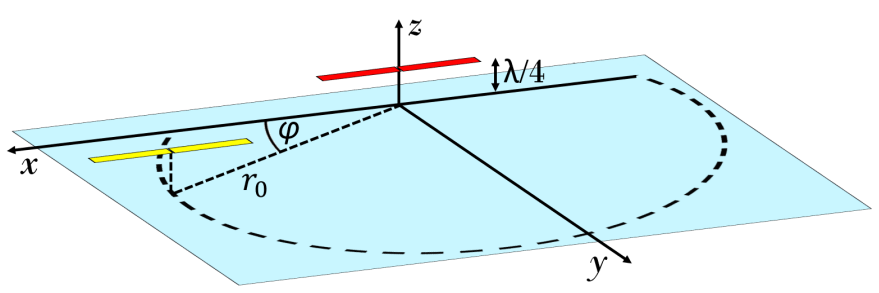

Fig. 4. Two-element array of $\lambda / 2$ strip dipoles in the xy-plane at $\lambda / 4$ above an infinite ground plane (light blue). The terminated element (yellow) is replaced around the excited element (red), and $r_{0}$ and $\varphi$ are the radial distance and angle between the elements, respectively.

While $\rho^{(q)}$ is used to analyze convergence numerically, the upper bound $\delta$ is used as stopping criterion. Note that the components of $\boldsymbol{W}^{(q)}$ could be weighted, especially when coupling levels vary strongly as in the case of large arrays or rapidly decreasing coupling.

\section{B. Simulation settings}

For numerical validation of our iterative method, the MoMcharacterization of an isolated element is performed in FEKO according to the guidelines summarized in Sec. II for the unitamplitude voltage source and a set of $\hat{\boldsymbol{\theta}}^{\prime}$ and $\hat{\phi}^{\prime}$-polarized incident plane waves with unit-amplitude $(1 \mathrm{~V} / \mathrm{m})$, with directional sampling per $10^{\circ}$. In correspondence with our description in Sec. II-B on the ground-plane model, a test case of two dipoles in half space is handled by directional sampling in both $\theta$ and $\phi$ to capture the 3D nature of the MC given the images of the dipoles. The other test cases can be handled by directional sampling in $\phi$ only with $\theta=90^{\circ}$, since they concern planar arrays with elements in free space or connected to a ground plane. The antennas are loaded with $Z_{0}=50 \Omega$ in the driven mode and terminated by the same impedance in the scattering mode. The generated far fields are turned into SWEs, which are truncated to capture the total power radiated into the far-field up to $0.01 \%$ at least. In our work, the number of modes is chosen the same for all radiated and scattered fields for the defined excitations. Cubic interpolation is applied to the expansion coefficients of the SWEs to get the SWEs for directions of incidence that are not simulated.

In the iteration process of our method, the stopping criterion (10) is used with $\delta=0.001$ for all S-parameter and field simulations. For numerical convergence analysis, an upper bound on the number of iterations is used. The results of our method are compared to the MoM/MLFMM of FEKO in which the automatic "fine" mesh setting is used. All simulations have been performed on an Intel i7-8650U quad-core processor at $1.9 \mathrm{GHz}$ with $32 \mathrm{~GB}$ of RAM. Moreover, our method is implemented in the programming language Julia [49].

\section{Numerical results}

To get a first insight in the behaviour of our iterative method, two dipoles in half space are considered, where not only their spacing but also their orientations are varied, as illustrated in Fig. 4. Only one dipole is excited. As measure of far-field

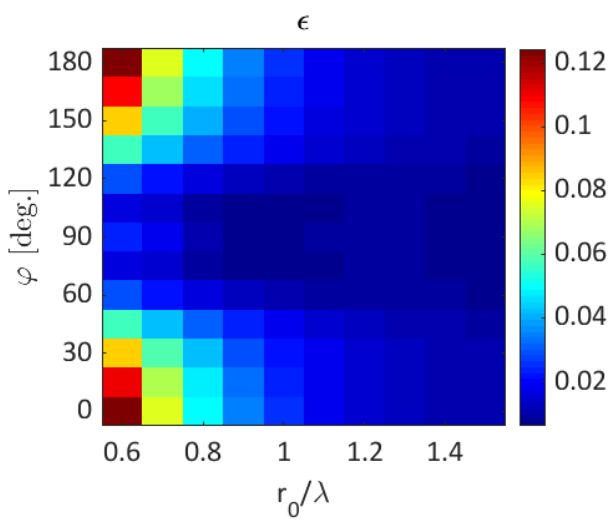

Fig. 5. Relative error $\epsilon$ of the embedded far field of two dipoles in a half space versus radial spacing $r_{0}$ and angle $\varphi$ defined in Fig. 4.

accuracy, the relative error $\epsilon$ is introduced by

$$
\epsilon=\frac{\sqrt{\iint_{\Omega}\left[\left|F_{\theta}^{\mathrm{J}}-F_{\theta}^{\mathrm{MoM}}\right|^{2}+\left|F_{\phi}^{\mathrm{J}}-F_{\phi}^{\mathrm{MoM}}\right|^{2}\right] d \Omega}}{\sqrt{\iint_{\Omega}\left[\left|F_{\theta}^{\mathrm{MoM}}\right|^{2}+\left|F_{\phi}^{\mathrm{MoM}}\right|^{2}\right] d \Omega}} .
$$

Here $F_{\theta}^{\mathrm{X}}$ and $F_{\phi}^{\mathrm{X}}$ are the far-field components obtained from our method $(\mathrm{X}=\mathrm{J})$ and FEKO $(\mathrm{X}=\mathrm{MoM})$, respectively, $\Omega$ represents the surface of the half sphere described in $(\theta, \phi)$, and $d \Omega=\sin \theta d \theta d \phi$. Fig. 5 shows $\epsilon$ versus the radial spacing $r_{0}$ between the elements and their angle $\varphi$ defined in Fig. 4. As expected the error gets more pronounced for decreasing radial separation $r_{0}$ at constant angle $\varphi$. The maximum error is observed for collinear dipole configurations $\varphi=\left\{0^{\circ}, 180^{\circ}\right\}$ and the smallest considered spacing of $0.6 \lambda$. In that case the neighbouring tips of the dipoles are not in each others far field, and the radial field component and radial dependence cannot be ignored presumably, which may explain the rise of the relative error.

The performance of our iterative method has been examined further on two types of arrays: a regular array of PIFAs and an ISA of log-periodic elements. Schematics of the PIFA array are depicted in Fig. 6, while Figs. 7 and 8 show the individual log-periodic element and the ISA of log-periodic elements, respectively. Next, S-parameters, embedded far-field patterns, and array patterns obtained for these arrays are compared with MoM/MLFMM results obtained by FEKO.

1) S-parameters and active reflection coefficients: Fig. 9 shows the comparison of the reflection parameters of the center element i $\left(S_{i i}\right)$ and the corner element $\mathrm{j}\left(S_{j j}\right)$ of the PIFA array of Fig. 6 over the frequency band of interest. The figure shows good matching between the results of our method and those of FEKO. The parameters of the isolated element are shown to illustrate the non-negligible effect of MC.

Fig. 10 shows the active reflection coefficients versus frequency for center and corner elements of the $11 \times 11$ PIFA array in the case of: (a) the uniform excitation and (b) scanning to $\theta_{s}=60^{\circ}$ in the plane $\phi_{s}=0^{\circ}$ by linear phase tapering. Despite slight deviations in amplitude and resonance peak, our results match those of FEKO reasonably well. It should be noted that, from a design point of view, the active reflection 


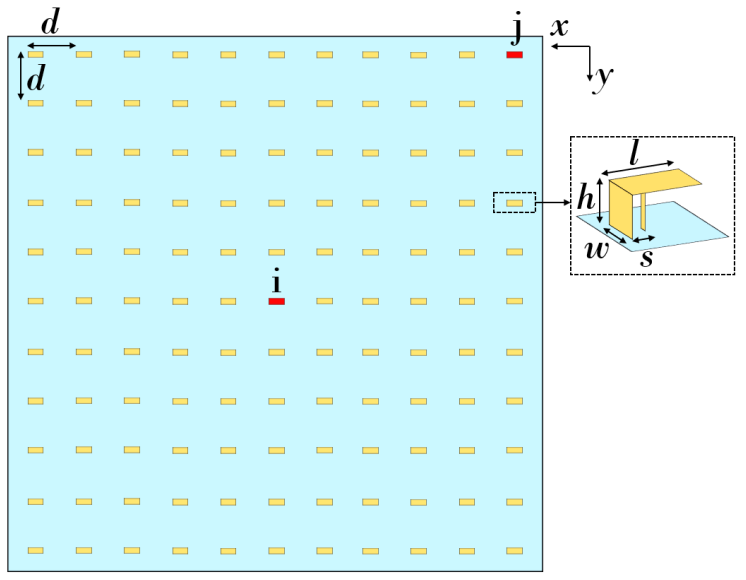

Fig. 6. Top view of an $11 \times 11$ regular PIFA array (with infinite ground plane in light blue): $f_{0}=28 \mathrm{GHz}, l=2.14 \mathrm{~mm}, w=0.5 \mathrm{~mm}, h=$ $0.71 \mathrm{~mm}, s=0.39 \mathrm{~mm}, d=0.829 \lambda_{0}=8.88 \mathrm{~mm}$. EEPs are computed for the center and corner elements labeled "i" and "j", respectively.

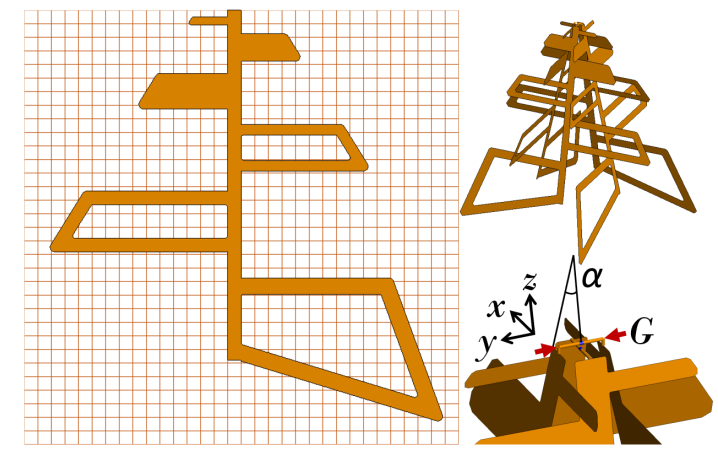

Fig. 7. A single element of the ISA of log-periodic elements shown in Fig. 8 The element (upper-right plot) consists of four identical metallic arms shown on the $1 \mathrm{~cm}$ grid (left plot), each arm tilted by $\alpha=12.5^{\circ}$ w.r.t. the $z$-axis. The element is fed through a wire port placed along the $x$-axis with $G=1.4$ $\mathrm{cm}$ and $Z_{0}=50 \Omega$ (lower-left plot).

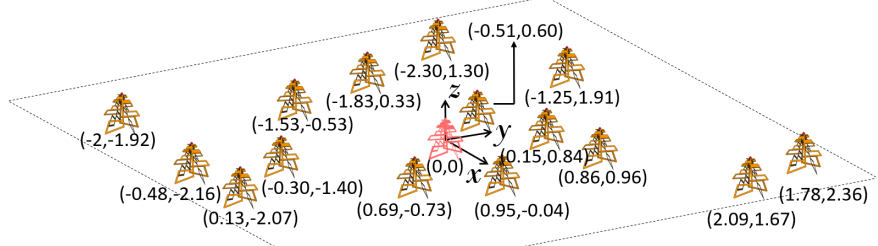

Fig. 8. 16-element ISA of log-periodic elements in free space with annotated element positions $(x, y)$ given in [m]. EEP is computed for the red-colored element.

of the center element is not favorable for broadside radiation and the scanned example exhibits multiple grating lobes due to the relatively large spacing of $d=0.829 \lambda_{0}$. Though the practical relevance may be limited for various applications, the numerical examples show particularly that our method approximates well active reflection parameters for different scanning conditions.

2) Embedded-element and array patterns: Fig. 11 shows the EEPs of the center ((a)-(b)) and corner ((c)-(d)) elements of the PIFA array in Fig. 6. The excitation of the reference
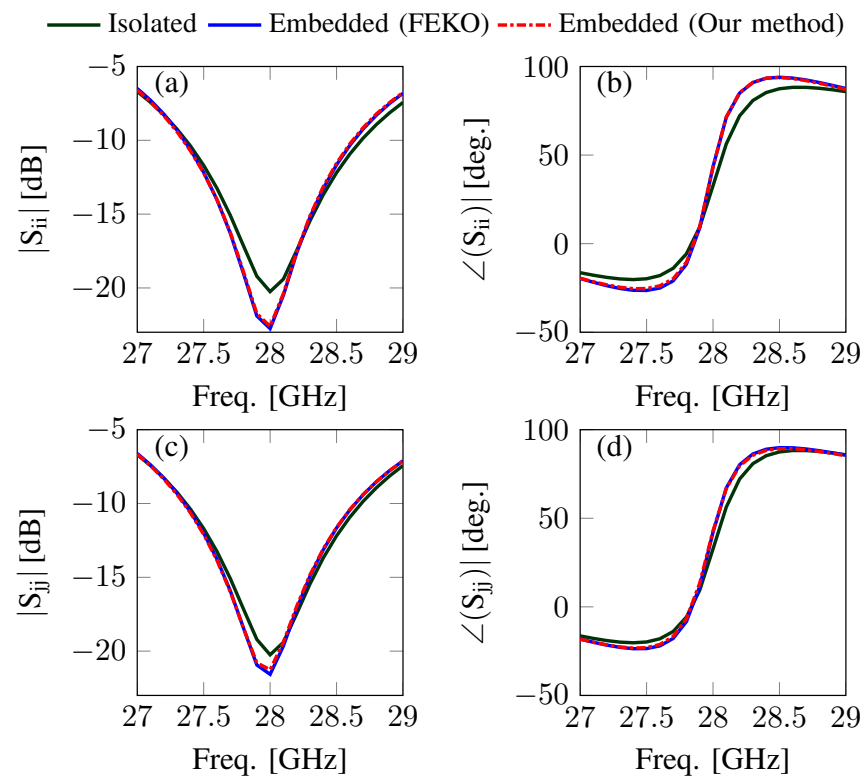

Fig. 9. $S_{\mathrm{ii}}$ and $S_{\mathrm{jj}}$ versus frequency for the center element $\mathrm{i}$ and the corner element $\mathrm{j}$ of the $11 \times 11$ PIFA array in Fig. 6 .

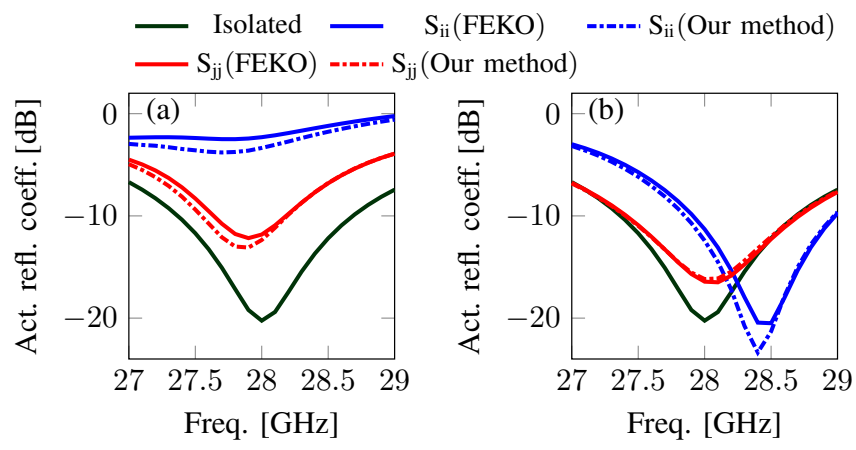

Fig. 10. Active reflection coefficients versus frequency for the center and corner elements $\mathrm{i}$ and $\mathrm{j}$ of the $11 \times 11$ PIFA array in Fig. 6: (a) uniformlyexcited array; (b) scanned array $\left(\theta_{s}=60^{\circ}, \phi_{s}=0^{\circ}\right)$.

elements is $1 \mathrm{~V}$. The plots labeled (a) and (c) show the field magnitude for the plane $\phi=0^{\circ}$ (xz-plane) while the plots labeled (b) and (d) show the field magnitude for $\theta=90^{\circ}$ (xyplane or plane of the array). As in the case of the reflection parameters, the figure shows good matching between the results of our iterative method and those of FEKO. In particular, our method captures rather well the highly oscillatory nature of the field of the PIFA array in the azimuth plane. Only in the top of the central peak in the elevation plane our method seems to underestimate the field amplitude. Note that Fig. 11 shows the isolated element pattern (IEP) to illustrate again the non-negligible effect of MC.

To illustrate the applicability of our method to ISAs, Fig. 12 shows the EEPs corresponding to the red-colored element of the 16-element ISA of log-periodic elements in Fig. 8. In this figure the EEPs with MC follow the IEP since the MC is lower, but it still induces a significant ripple as can be appreciated in 

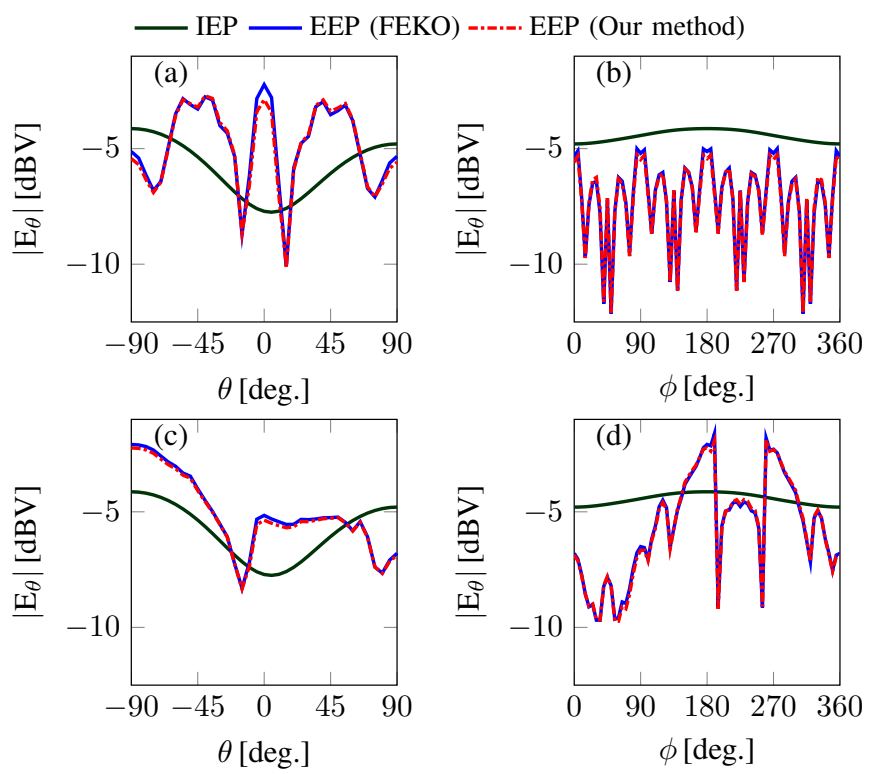

Fig. 11. EEP of the center element i (a)-(b), and the corner element j (c)-(d) of the $11 \times 11$ PIFA array in Fig. 6 at $f_{0}=28 \mathrm{GHz}$ at the $\phi=0^{\circ}$ (left plots) and $\theta=90^{\circ}$ planes (right plots).
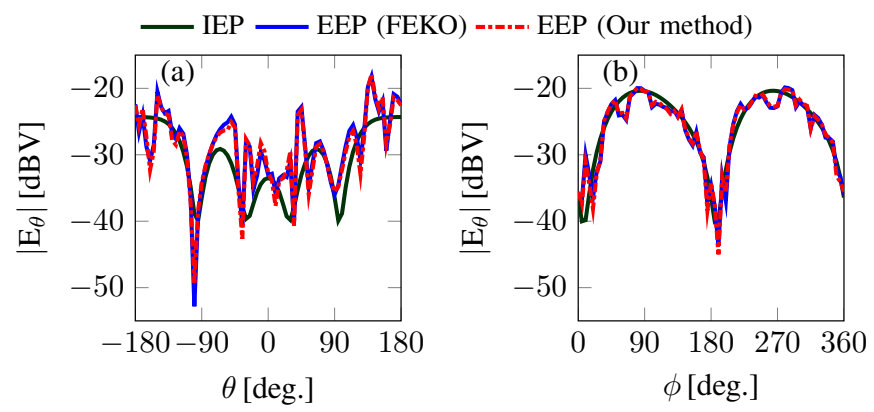

Fig. 12. EEP of the red-colored element of the 16-element ISA of log-periodic elements in Fig. 8 at $f_{0}=600 \mathrm{MHz}$ : (a) plane $\phi=0^{\circ}$; (b) plane $\theta=90^{\circ}$.

the results of both FEKO and our method.

Finally, to illustrate the performance of our method when all elements are excited with uniform phase and amplitude, Fig. 13 (a) shows the amplitude of the far-field pattern of the PIFA array in the plane $\phi=0^{\circ}$ with all elements excited by $1 \mathrm{~V}$. Similarly, Fig. 13 (b) shows the phase in the plane $\theta=90^{\circ}$. The peaks in amplitude and the oscillations in phase are well-reproduced by our method.

3) Convergence and parameter dependence: To get insight in convergence and spacing dependence, the relative difference $\rho^{(q)}$ in (10) is shown in Fig. 14 as function of the iteration index $q$ for the $11 \times 11$ PIFA array with 10 different spacing values, where center element $\mathrm{i}$ is excited at $f_{0}=28 \mathrm{GHz}$. All descending curves show linear dependence of $\log \rho^{(q)}$, which indicates that the method converges exponentially. This numerical result demonstrates consistency of our multiplescattering inspired method with classical theory of Neumann
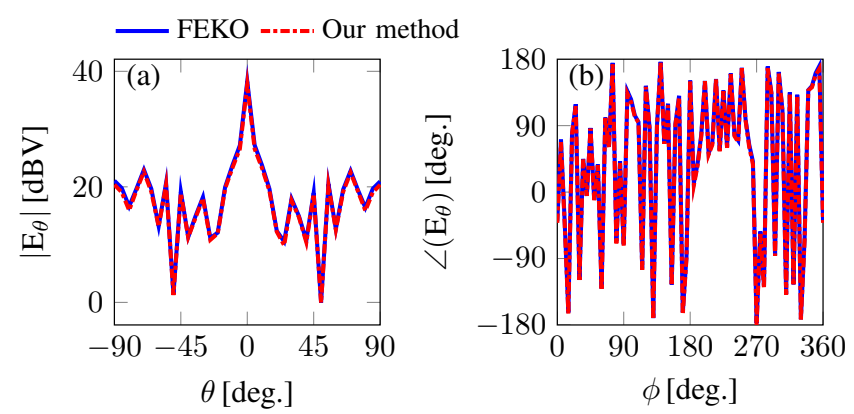

Fig. 13. Far-field pattern of the $11 \times 11$ PIFA array in Fig. 6 at $f_{0}=28 \mathrm{GHz}$ and an excitation of $1 \mathrm{~V}$ on all elements: (a) amplitude in the plane $\phi=0^{\circ}$; (b) phase in the plane $\theta=90^{\circ}$.

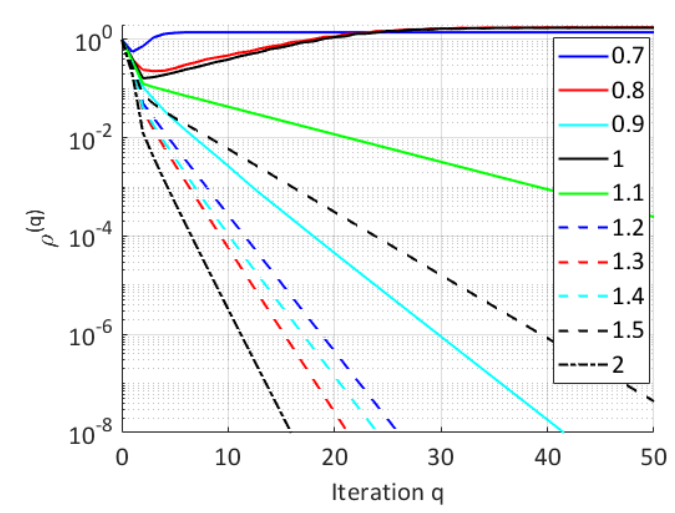

Fig. 14. Convergence plot for the Jacobi scheme (4): the relative difference $\rho^{(q)}$ in (10) versus iteration index $q$ in case the center element $\mathrm{i}$ of the $11 \times 11$ PIFA array in Fig. 6 is excited at $f_{0}=28 \mathrm{GHz}$. The legend lists spacing in terms of $\lambda_{0}$.

series [47, Theorem 8.32] on which the Jacobi method is based. Specifically, for the decomposed MoM matrix at the beginning of Sec. II-A, the logarithm of the error term of truncating that series is $q \log \left\|\boldsymbol{Z}_{\text {on }}^{-1} \boldsymbol{Z}_{\text {off }}\right\|$. The slope varies with the coupling level described by $\boldsymbol{Z}_{\text {off }}$ and, hence, with the spacing. Similar slope variation can be observed in Fig. 14. The case $1.5 \lambda$ converges slower than the cases 1.2 to $1.4 \lambda$, and the case $\lambda$ does not converge, while both the $0.9 \lambda$ and $1.1 \lambda$ cases converge. In this respect, we note that multiples of $d=0.5 \lambda$ are special cases due to the (approximate) inphase and out-of-phase interference along rows and columns of the array. Moreover, the non-convergent behaviour for $d=\lambda$ coincides with the first grating lobe being excited along the array plane.

The convergence rate and robustness of our method can be improved by adopting a more robust Gauss-Seidel-based iterative scheme instead of a Jacobi-based scheme. The GaussSeidel method differs from the Jacobi method in that it uses the already available values from the $q$-th iteration to update the solution. Consequently, the iterative scheme (4) turns into

$$
\underline{\boldsymbol{w}}_{p m}^{(q)}=\left.\underline{\boldsymbol{E}}_{m}^{(0)}\right|_{\theta, \phi}+\left.\sum_{\substack{m^{\prime}=1 \\ m^{\prime} \neq m}}^{N}\left(\underline{\underline{\boldsymbol{E}}}_{m m^{\prime}}^{\mathrm{s}(0)} \underline{\boldsymbol{w}}_{m m^{\prime}}^{\left(q_{s}\right)}\right)\right|_{\theta, \phi},
$$




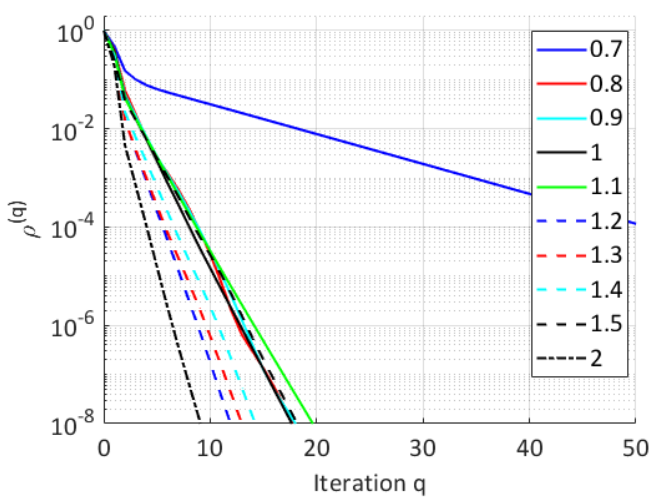

Fig. 15. Convergence plot for the Gauss-Seidel scheme (12): the relative difference $\rho^{(q)}$ in (10) versus iteration index $q$ in case the center element $\mathrm{i}$ of the $11 \times 11$ PIFA array in Fig. 6 is excited at $f_{0}=28 \mathrm{GHz}$. The legend lists spacing in terms of $\lambda_{0}$.

where

$$
q_{s}= \begin{cases}q, & m<p \\ q-1, & m>p .\end{cases}
$$

According to classical theory, the Gauss-Seidel method converges in case $\left\|\left(\boldsymbol{Z}_{\text {on }}+\boldsymbol{Z}_{\text {off, } \mathrm{L}}\right)^{-1} \boldsymbol{Z}_{\text {off, }}\right\|<1$ for any norm, where the subscripts $L$ and $U$ denote the lower and upper triangular parts of $\boldsymbol{Z}_{\text {off. }}$. Similar to the classical theory of the Jacobi method described in Subsec. III-A, a necessary and sufficient condition is obtained by replacing the norm by the spectral radius. Moreover, like the Jacobi method, the GaussSeidel method converges for strictly dominant or irreducibly diagonally dominant matrices $Z$ [48, Theorem 3.10]. The advantages of using the Gauss-Seidel-based iterative scheme over the Jacobi-based scheme are demonstrated in Fig. 15 for the $11 \times 11$ PIFA array in Fig. 14. Clearly, the Gauss-Seidelbased scheme shows convergence for all spacing values as well as improved convergence rates. To illustrate the convergence rate for an element which is electrically speaking relatively large, the relative difference $\rho^{(q)}$ is plotted in Fig. 16 versus iteration index $q$ for an $11 \times 11$ array of the log-periodic elements in Fig. 7. Ten different spacing values are considered, the center element $\mathrm{i}$ is excited at $f_{0}=1 \mathrm{GHz}$, and the GaussSeidel based scheme is employed. Clearly, slower convergence rates are realized as compared to the results for the PIFA array in Fig. 15. Improved convergence may be expected for ISAs of electrically large elements instead of regular arrays of such elements. ISAs have generally larger spacing and their irregularity leads to incoherence in coupling, which mitigates convergence issues. One might also consider implementing a typically more robust Krylov subspace iterative technique, possibly coupled with a preconditioner aimed at improving the convergence, in what would be inspired by classical preconditioning for iterative methods applied to linear systems of equations or by specific solutions for MoM systems such as the MBF and CBFM formulations mentioned in the introduction.

The Jacobi and Gauss-Seidel schemes yield, up to the requested numerical accuracy, the same S-parameter and farfield results in case both schemes converge. For the smallest

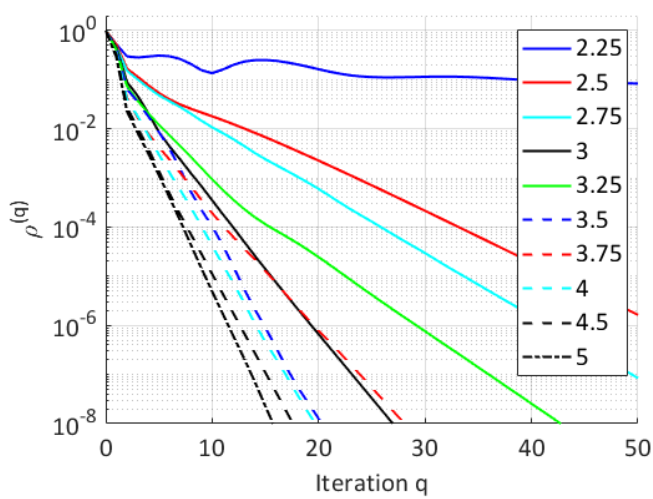

Fig. 16. Convergence plot for the Gauss-Seidel scheme (12): the relative difference $\rho^{(q)}$ in (10) versus iteration index $q$ in case the center element of the regular $11 \times 11$ array of log-periodic elements shown in Fig. 7 is excited at $f_{0}=1 \mathrm{GHz}$. The legend lists spacing in terms of $\lambda_{0}$.

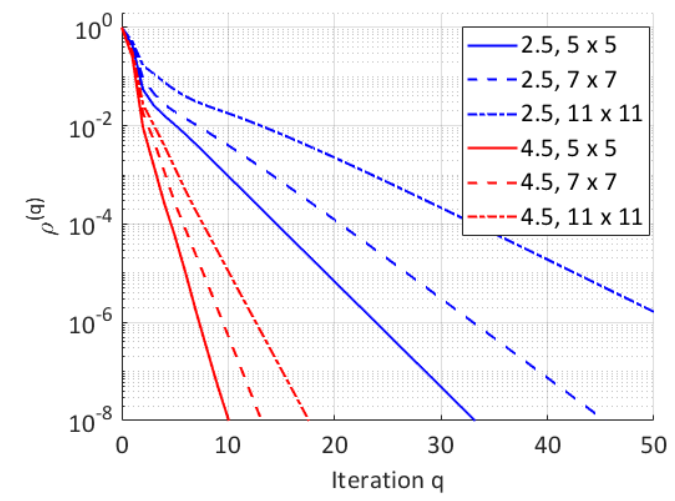

Fig. 17. Convergence plot for the Gauss-Seidel scheme (12): the relative difference $\rho^{(q)}$ in (10) versus iteration index $q$ in case the center element of regular arrays of log-periodic elements shown in Fig. 7 is excited at $f_{0}=$ $1 \mathrm{GHz}$. The legend lists spacing in terms of $\lambda_{0}$ and array size.

spacing values considered above, such as $0.7 \lambda$ for the PIFA array, only the Gauss-Seidel scheme converges and its Sparameter and far-field results compare reasonably well to fullwave simulations by FEKO. For even smaller spacing values, the scheme may still converge, but the accuracy of the solution deteriorates. Further research is necessary to see whether this can be remedied by modifications of the approximations in the method, as described in the conclusion (Sec. IV).

The convergence rates seem only weakly dependent on array size and excitation type (center or corner element, or uniform amplitude taper with or without scanning by a linear phase taper). The weak dependence on excitation is not that surprising given that, in case a single element is excited, one can change its source amplitude and phase in the iteration scheme (4) without affecting $\rho^{(q)}$ in (10). Other variations of excitation (source at a different element or multiple elements with sources) lead to a slightly different weighting of error terms in $\rho^{(q)}$, which mainly affects accuracy (for a fixed number of iterations). The dependence on array size is illustrated in Fig. 17 for three sizes of the array of log-periodic elements and two spacing values. The convergence rate decreases somewhat with array size, though the exact dependence is difficult to 
discern (spacing plays a significant role).

\section{Computational complexity}

In this section the computational complexity and run time of our method are discussed and compared to the standard MoM solver in FEKO as well as its iterative MLFMM/BiCGSTAB solver. As described in the introduction the complexity of the MLFMM is $\mathcal{O}\left(N_{\mathrm{x}} \log ^{2} N_{\mathrm{x}}\right)$ for large numbers of basis functions $N_{\mathrm{x}}$. A single iteration of our method scales as $\mathcal{O}\left(N^{3}\right)$ for large numbers of elements $N$, which directly follows from the iteration scheme (4) with $N^{2}$ unknowns and a summation of $N$ terms per unknown. This scaling is also applicable to the complete iteration process, if the number of iterations is fixed (regardless of $N$ ) or the (exponential) convergence rate depends weakly on $N$ as numerically observed. From the orders of complexity, it is clear that our method will be at its best, as compared to MLFMM, for medium-sized arrays up to hundreds of elements and a complex single element that requires many basis functions such that $N_{\mathrm{x}}$ on the total array is much bigger than $N$. In such cases the computational time and the memory use of MLFMM or standard MoM applied to the total array can become huge, especially when the array lattice lacks symmetry, as will be shown for the ISA of log-periodic elements. The run time of our method for the PIFA array used in the validation will be less outstanding as compared to fullwave simulation of the complete array, since the elements are relatively simple and the regularity of the array lattice can be used to reduce the effort of the MC computation.

To asses the run times of our method, its total computation time is decomposed into three main constituents:

- "Isolated element": time required to compute the radiated and scattered fields of a single isolated element for the voltage source and all plane-wave excitations (36 for all three validation examples) by FEKO.

- "SWE": time required to compute the SWE of a set of radiated and scattered far-fields of the isolated element. Note that the modal truncation indices $n$ in the SWE expansions are 3 and 8 for the PIFA and log-periodic elements, respectively.

- "Array (our method)": time required to compute converged weight vectors for all defined array excitations.

Recall that the isolated element characterization and the SWE need to be computed only once, while the iteration process needs to be run for every array excitation and frequency. For the simulations of complete arrays with FEKO, only the total time will be listed of either its standard MoM solver with LU decomposition or its MLFMM/BiCGSTAB solver.

For the regular PIFA arrays with the spacing $d=0.829 \lambda_{0}$, the run times are listed in Table I for the computation of all EEPs. For FEKO the times of its standard MoM are listed for the following reason. While an iterative method needs to be run for each of array excitation separately, the inversion in a standard MoM needs to be carried out only once after which calculating the response for each excitation is a matter of matrix multiplications. Hence, for large numbers of excitations, a standard MoM may be preferred as compared to an iterative solver. In our comparison the number of different
TABLE I

RUN TIMES FOR ALL EEPS (HR:MIN:S) OF $N$-ELEMENT PIFA ARRAYS WITH PARAMETER VALUES AS IN FIG. 6

\begin{tabular}{|ccccc|}
\hline$N$ & $\begin{array}{c}\text { Isolated elem. } \\
\text { (FEKO MoM) }\end{array}$ & SWE & $\begin{array}{c}\text { Array } \\
\text { (our method) }\end{array}$ & $\begin{array}{c}\text { Array } \\
(\text { FEKO MoM) }\end{array}$ \\
\hline $5 \times 5$ & $00: 00: 04$ & $00: 00: 01$ & $00: 00: 02$ & $00: 04: 07$ \\
$7 \times 7$ & $00: 00: 04$ & $00: 00: 01$ & $00: 00: 10$ & $00: 12: 03$ \\
$9 \times 9$ & $00: 00: 04$ & $00: 00: 01$ & $00: 01: 07$ & $00: 37: 09$ \\
$11 \times 11$ & $00: 00: 04$ & $00: 00: 01$ & $00: 04: 26$ & $01: 39: 18$ \\
\hline
\end{tabular}

TABLE II

RUN TIMES FOR A SINGLE EEP (HR:MIN:S) OF $N$-ELEMENT IRREGULAR ARRAYS OF LOG-PERIODIC ELEMENTS AS ILLUSTRATED IN FIG. 8.

\begin{tabular}{|ccccc|}
\hline$N$ & $\begin{array}{c}\text { Isolated elem. } \\
\text { (FEKO } \\
\text { MLFMM) }\end{array}$ & SWE & $\begin{array}{c}\text { Array } \\
\text { (our method) }\end{array}$ & $\begin{array}{c}\text { Array } \\
\text { (FEKO } \\
\text { MLFMM) }\end{array}$ \\
\hline 8 & $00: 53: 39$ & $00: 00: 04$ & $00: 00: 02$ & $04: 14: 37$ \\
16 & $00: 53: 39$ & $00: 00: 04$ & $00: 00: 08$ & $07: 21: 40$ \\
\hline
\end{tabular}

excitations or EEPs is 25 or more and therefore the standard MoM times are listed. The array run times of our method scale approximately with $N^{4}$ (the complexity for computing all EEPs), though not exactly since they depend also on the number of iterations, which in turn depends on the setting of the threshold $\delta$. The array run times of FEKO's standard MoM seem to scale as $N_{\mathrm{x}}^{2}$, since the matrix assembly still dominates, in terms of numerical effort, the LU decomposition for relatively small array sizes. As expected for relatively simple elements in regular lattices, the use of our method provides only small to moderate run time benefits as compared to FEKO. Though, we should note that if the spacing or element location is varied instead of the excitation, for example in an optimization scheme, the trade-off is significantly more favorable for our method, since in that case both our method and the MoM method need to be carried out for every spacing.

A different picture arises when considering the irregular array of log-periodic elements. Since only a single excitation or EEP is considered (the one indicated in Fig. 8), it is fair to compare with FEKO's MLFMM/BiCGSTAB solver. As Table II shows, that solver requires a gargantuan computational effort for even a small number of elements, while the effort of our method is rather modest. Additionally, a twice as large array takes about 4 and 1.7 times more time for our method and FEKO's MLFMM/BiCGSTAB, respectively. These factors deviate from the expected scaling of $N^{3}$ and $N_{\mathrm{x}} \log ^{2} N_{\mathrm{x}}$, but this deviation may be due to the limited array sizes. Aspects of e.g. initialization may still be significant in terms of CPU times, particularly for our method with CPU times of order seconds. When the logged times are extrapolated to arrays of larger size, it is clear that our method will outperform the MLFMM, as expected, for similar mediumsized irregular arrays of log-periodic elements up to hundreds of elements. For more complex elements with more basis functions per element, the limit in array size will grow. Additionally, the computational effort of our method could be reduced by several means. Firstly, by defining a radius of influence for the MC, the number of terms to sum in (4) could be reduced. However, since several small contributions may sum up to a significant contribution, the added value may be 
limited. Along similar lines, one could limit the number of iterations for unknowns $\underline{\boldsymbol{w}}_{p m}^{(q)}$ between distant elements $p$ and $m$. Possibly, the performance of our method could benefit from the relaxation ideas discussed in [50, Ch. 4.2.1].

\section{CONCLUSION}

In this paper we have detailed a method for efficient estimation of antenna array mutual coupling based on the concept of multiple scattering and the availability of far-field data of an isolated element for different types of excitation, i.e., local source(s) and plane waves with different directions. These data can be obtained from any full-wave simulator. Mutual interactions between the array elements are modeled by approximating the incident field as a pair of plane waves with orthogonal polarization taken from the spherical wave expansion of propagating field from one element to another. The resulting iteration scheme relates to the iterative Jacobi and Gauss-Seidel methods for the solution of linear systems of equations and shows similar convergence behaviour as numerically demonstrated. The method has been tested for regular arrays of PIFA elements and irregular sparse arrays of log-periodic elements, and shows good agreement with full-wave simulations on the complete array in terms of the embedded element patterns and S-parameters. Significant runtime savings have been observed for small to medium sized arrays compared to a direct MoM solver and an iterative solver using MLFMM and BiCGSTAB. Further research directions are: using near-field data and adding the contribution of the radial component to the SW-PW described coupling between elements, in order to improve the applicability of the method to arrays with relatively small spacing.

\section{ACKNOWLEDGMENT}

This project has received funding from the European Union's Horizon 2020 research and innovation program under the Marie Skłodowska-Curie grant agreement No. 721732. The study is based on the research supported in part by the National Research Foundation of South Africa (Grant Numbers: 75322 and 106033).

\section{REFERENCES}

[1] H. Bui-Van, J. Abraham, M. Arts, Q. Gueuning, C. Raucy, D. GonzálezOvejero, E. de Lera Acedo, and C. Craeye, "Fast and accurate simulation technique for large irregular arrays," IEEE Trans. Antennas Propag., vol. 66, no. 4, pp. 1805-1817, Apr. 2018.

[2] E. Björnson, L. Sanguinetti, H. Wymeersch, J. Hoydis, and T. L. Marzetta, "Massive MIMO is a reality-What is next? Five promising research directions for antenna arrays," Digit. Signal Process., vol. 94, pp. 3 - 20, Nov. 2019, special Issue on Source Localization in Massive MIMO.

[3] X. Ge, R. Zi, H. Wang, J. Zhang, and M. Jo, "Multi-user massive MIMO communication systems based on irregular antenna arrays," IEEE Trans. Wireless Commun., vol. 15, no. 8, pp. 5287-5301, Aug. 2016.

[4] G. Toso, C. Mangenot, and A. G. Roederer, "Sparse and thinned arrays for multiple beam satellite applications," in 2nd European Conf. Antennas Propag., EuCAP 2007, Nov. 2007, pp. 1-4.

[5] M. P. van Haarlem, M. W. Wise, A. W. Gunst, G. Heald, J. P. McKean et al., "LOFAR: The LOw-Frequency ARray," Astronomy and Astrophysics, vol. 556, pp. A2, 1-53, Jul. 2013.

[6] C. Bencivenni, M. V. Ivashina, R. Maaskant, and J. Wettergren, "Synthesis of maximally sparse arrays using compressive sensing and full-wave analysis for global Earth coverage applications," IEEE Trans. Antennas Propag., vol. 64, no. 11, pp. 4872-4877, Nov. 2016.
[7] — "Design of maximally sparse antenna arrays in the presence of mutual coupling," IEEE Antennas Wireless Propag. Lett., vol. 14, pp. 159-162, 2015.

[8] H. V. Bui, S. N. Jha, and C. Craeye, "Fast full-wave synthesis of printed antenna arrays including mutual coupling," IEEE Trans. Antennas Propag., vol. 64, no. 12, pp. 5163-5171, Dec. 2016.

[9] J. I. Echeveste, M. Á. González de Aza, J. Rubio, and C. Craeye, "Gradient-based aperiodic array synthesis of real arrays with uniform amplitude excitation including mutual coupling," IEEE Trans. Antennas Propag., vol. 65, no. 2, pp. 541-551, Feb. 2017.

[10] C. Craeye and D. González-Ovejero, "A review on array mutual coupling analysis," Radio Sci., vol. 46, RS2012, no. 2, pp. 1-25, Apr. 2011.

[11] F. Gustrau and D. Manteuffel, EM Modeling of Antennas and RF Components for Wireless Communication Systems. Berlin and Heidelberg: Springer-Verlag, 2006.

[12] A. Vasylchenko, Y. Schols, W. De Raedt, and G. A. E. Vandenbosch, "A benchmarking of six software packages for full-wave analysis of microstrip antennas," in 2nd European Conf. Antennas Propag., EuCAP 2007, Nov. 2007, pp. 1-6.

[13] A. Ishimaru, R. Coe, G. Miller, and W. Geren, "Finite periodic structure approach to large scanning array problems," IEEE Trans. Antennas Propag., vol. AP-33, no. 11, pp. 1213-1220, Nov. 1985.

[14] J. Song, C.-C. Lu, and W. C. Chew, "Multilevel fast multipole algorithm for electromagnetic scattering by large complex objects," IEEE Trans. Antennas Propag., vol. 45, no. 10, pp. 1488-1493, Oct. 1997.

[15] Ö. Ergül and L. Gürel, The Multilevel Fast Multipole Algorithm (MLFMA) for Solving Large-Scale Computational Electromagnetics Problems, ser. IEEE Press Series on Electromagnetic Wave Theory, A. C. Cangellaris, Ed. Piscataway, NJ, USA: John Wiley and Sons, Ltd, 2014.

[16] D. Ludick, U. Jakobus, and D. B. Davidson, "Efficient analysis of finite antenna arrays using the domain Green's function method," in Proc. 2012 IEEE Int. Symp. Antennas Propag., Jul. 2012, pp. 1-2.

[17] E. Suter and J. Mosig, "A subdomain multilevel approach for the efficient MoM analysis of large planar antennas," IEEE Microw. Opt. Technol. Lett., vol. 26, no. 4, pp. 270 - 277, Aug. 2000.

[18] L. Matekovits, V. A. Laza, and G. Vecchi, "Analysis of large complex structures with the synthetic-functions approach," IEEE Trans. Antennas Propag., vol. 55, no. 9, pp. 2509-2521, Sep. 2007.

[19] L. Matekovits, G. Vecchi, M. Bercigli, and M. Bandinelli, "Syntheticfunctions analysis of large aperture-coupled antennas," IEEE Trans. Antennas Propag., vol. 57, no. 7, pp. 1936-1943, Jul. 2009.

[20] E. Lucente, G. Tiberi, A. Monorchio, G. Manara, and R. Mittra, "The characteristic basis function method (CBFM): A numerically efficient strategy for solving large electromagnetic scattering problems," Turkish J. Electr. Eng., vol. 16, no. 1, pp. 41-56, 2008.

[21] R. Maaskant, R. Mittra, and A. Tijhuis, "Fast analysis of large antenna arrays using the characteristic basis function method and the adaptive cross approximation algorithm," IEEE Trans. Antennas Propag., vol. 56, no. 11, pp. 3440-3451, Nov. 2008.

[22] G. Allaire and S. M. Kaber, Numerical Linear Algebra, ser. Texts in Applied Mathematics 55, J. Marsden, L. Sirovich, and S. Antman, Eds. New York, NY, USA: Springer, 2008.

[23] "Numerical methods in FEKO," White Paper, Altair Engineering, Inc., Dec. 2016.

[24] M. Fruhnert, I. Fernandez-Corbaton, V. Yannopapas, and C. Rockstuhl, "Computing the T-matrix of a scattering object with multiple plane wave illuminations," Beilstein J. Nanotechnol., vol. 8, p. 614—626, Mar. 2017.

[25] J. Rubio, M. A. González, and J. Zapata, "Generalized-scattering-matrix analysis of a class of finite arrays of coupled antennas by using 3-D FEM and spherical mode expansion," IEEE Trans. Antennas Propag., vol. 53, no. 3, pp. 1133-1144, Mar. 2005.

[26] H. A. Abdallah and W. Wasylkiwskyj, "A numerical technique for calculating mutual impedance and element patterns of antenna arrays based on the characteristics of an isolated element," IEEE Trans. Antennas Propag., vol. 53, no. 10, pp. 3293-3299, Oct. 2005.

[27] S. Lou, B. Duan, W. Wang, C. Ge, and S. Qian, "Analysis of finite antenna arrays using the characteristic modes of isolated radiating elements," IEEE Trans. Antennas Propag., vol. 67, no. 3, pp. 15821589, Mar. 2019

[28] A. M. van de Water, "LEGO - linear embedding via Green's operators," $\mathrm{Ph} . \mathrm{D}$. dissertation, Eindhoven University of Technology, Eindhoven, 2007. [Online]. Available: http://alexandria.tue.nl/extra2/200711874.pdf

[29] F. J. Demuynck, G. A. E. Vandenbosch, and A. R. Van de Capelle, "The expansion wave concept-Part I: Efficient calculation of spatial Green's functions in a stratified dielectric medium," IEEE Trans. Antennas Propag., vol. 46, no. 3, pp. 397-406, Mar. 1998. 
[30] G. A. E. Vandenbosch and F. J. Demuynck, "The expansion wave concept-Part II: A new way to model mutual coupling in microstrip arrays," IEEE Trans. Antennas Propag., vol. 46, no. 3, pp. 407-413, Mar. 1998.

[31] C.-T. Tai, "An iterative method of solving a system of linear equations and its physical interpretation from the point of view of scattering theory," IEEE Trans. Antennas Propag., vol. 18, no. 5, pp. 713-714, Sep. 1970.

[32] S. Laxpati, "Comments on "An iterative method of solving a system of linear equations and its physical interpretation from the point of view of scattering theory"," IEEE Trans. Antennas Propag., vol. 19, no. 4, p. 566, Jul. 1971.

[33] A. T. Adams, J. Perini, J. R. Stewart, and T. E. Baldwin, Jr., "Numerical techniques for radiation and coupling problems," in 1973 IEEE Int Electromagn. Compat. Symp. Rec., Jun. 1973, pp. 219-225.

[34] Y. Brand, A. K. Skrivervik, J. R. Mosig, and F. E. Gardiol, "New iterative integral equation technique for multilayered printed array antennas," in MMET Conf. Proc. 1998 Int. Conf. Math. Methods Electromagn. Theory. MMET 98 (Cat. No.98EX114), vol. 2, Jun. 1998, pp. 615-617.

[35] A. C. Polycarpou, "Evaluation of stationary block iterative techniques for the solution of finite arrays using the FE-BI method and domain decomposition," in 1st European Conf. Antennas Propag., EuCAP 2006, Nov. 2006, pp. 1-6.

[36] H. Bui-Van, T. Pairon, and C. Craeye, "Fast iterative techniques for the simulations of large antenna arrays," in 2018 Int. Conf. Electromagn. Adv. Appl. (ICEAA), Sep. 2018, pp. 247-249.

[37] C. Geuzaine, A. Vion, R. Gaignaire, P. Dular, and R. V. Sabariego, "An amplitude finite element formulation for multiple-scattering by a collection of convex obstacles," IEEE Trans. Magn., vol. 46, no. 8, pp. 2963-2966, Aug. 2010.

[38] A. Vion, R. V. Sabariego, and C. Geuzaine, "A model reduction algorithm for solving multiple scattering problems using iterative methods," IEEE Trans. Magn., vol. 47, no. 5, pp. 1470-1473, May 2011.

[39] D. J. Ludick, M. M. Botha, R. Maaskant, and D. B. Davidson, "The CBFM-enhanced Jacobi method for efficient finite antenna array analysis," IEEE Antennas Wireless Propag. Lett., vol. 16, pp. 2700-2703, 2017.

[40] D. I. L. de Villiers and R. Maaskant, "Element pattern prediction in mutually-coupled arrays through isolated antenna characterization," in 2017 Int. Symp. Antennas Propag. (ISAP), Oct. 2017, pp. 1-2.

[41] J. A. Dobrowolski, Introduction to Computer Methods for Microwave Circuit Analysis and Design. Boston, London: Artech House, 1991.

[42] K. Pontoppidan, "GRASP technical description," TICRA, Copenhagen, Denmark, Tech. Rep., Jun. 2015.

[43] F. Jensen and A. Frandsen, "On the number of modes in spherical wave expansions," in 26th Annu. Meeting Symp. Antenna Meas. Tech. Assoc. (AMTA), Oct. 2004, pp. 489-494.

[44] C. Cappellin, O. Breinbjerg, and A. Frandsen, "Properties of the transformation from the spherical wave expansion to the plane wave expansion," Radio Sci., vol. 43, RS1012, no. 1, pp. 1-16, Feb. 2008.

[45] C. Scott, Modern Methods of Reflector Antenna Analysis and Design. Boston, London: Artech House, 1990.

[46] J. Fridén and G. Kristensson, "Calculation of antenna radiation center using angular momentum," IEEE Trans. Antennas Propag., vol. 61, no. 12, pp. 5923-5930, Dec. 2013.

[47] T. Lyche. (2013, Jul.) Lecture Notes for MAT-INF 4130. [Online]. Available: https://www.uio.no/studier/emner/matnat/math/ MAT-INF4130/h13/book2013.pdf

[48] R. S. Varga, Matrix Iterative Analysis, 2nd ed., ser. Springer Series in Computational Mathematics 27, R. Bank, R. L. Graham, J. Stoer, R. Varga, and H. Yserentant, Eds. Berlin and Heidelberg: SpringerVerlag, 2000.

[49] J. Bezanson, A. Edelman, S. Karpinski, and V. B. Shah, "Julia: A fresh approach to numerical computing," SIAM Rev., vol. 59, no. 1, pp. 65-98, 2017.

[50] A. Quarteroni, R. Sacco, and F. Saleri, Numerical Mathematics, ser. Texts in Applied Mathematics 37, J. E. Marsden, L. Sirovich, M. Golubitsky, and W. Jäger, Eds. New York, Berlin, Heidelberg: SpringerVerlag, 2000.

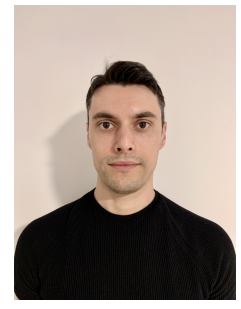

Tomislav Marinović (M'17) received the M.Sc. degree (summa cum laude) in electrical engineering from the University of Zagreb, Zagreb, Croatia, in 2016. Since Dec. 2016 he is a double-degree $\mathrm{PhD}$ candidate at $\mathrm{KU}$ Leuven, Leuven, Belgium and Chalmers University of Technology, Gothenburg, Sweden, working on the Marie Curie "SILIKA" project funded by the European Union's Horizon 2020 research and innovation programme. Within his $\mathrm{PhD}$, he was a Visiting Researcher at NXP and Eindhoven University of Technology, both Eindhoven, The Netherlands, TNO, The Hague, The Netherlands, Ericsson Research, Gothenburg, Sweden, and University of Stellenbosch, Stellenbosch, South Africa. His current research interests include antenna array modeling and antenna technologies for mobile communications.

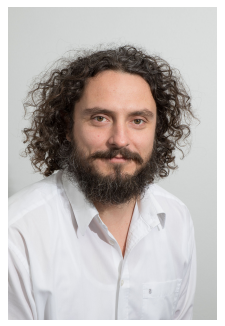

Dirk de Villiers (S'05-M'08-SM'15) received the B.Eng and Ph.D. degrees in electrical and electronic engineering from the University of Stellenbosch, Stellenbosch, South Africa in 2004 and 2007 respectively. From 2008 to 2009 he was a post-doctoral fellow at the University of Stellenbosch working on antenna feeds for the South African SKA program. He is currently a Professor at Stellenbosch University, where he holds the SARChI Research Chair in Antenna Systems for SKA. He has spent several months as visiting researcher at Antwerp University in Antwerp, Belgium and at Chalmers University of Technology in Gothenburg, Sweden. Since 2010 he has regularly worked on contract for EMSS Antennas (Pty) Ltd in Stellenbosch, South Africa, on the design of the reflector antenna systems for the MeerKAT, SKA, and ngVLA radio telescopes. His main research interests include reflector antennas for radio astronomy, as well as surrogate-based optimization of microwave components. $\mathrm{He}$ served as chair of the South African IEEE joint AP/MTT/EMC chapter between 2017-2018, and is a senior member of URSI.

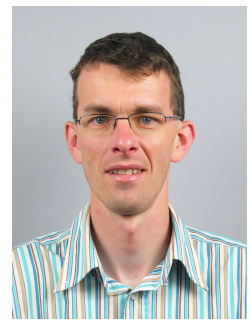

Dave J. Bekers (M'05, SM'17) received the M.Sc. degree in mathematics, P.D.Eng. degree, and Ph.D. degree from the Technische Universiteit Eindhoven, Eindhoven, The Netherlands, in 1999, 2001, and 2004, respectively. He carried out his final project for the postgraduate program "Mathematics for Industry" and his Ph.D. project with Thales Nederland, Hengelo, The Netherlands, in the field of array antennas. Since November 2004, he has been with TNO, the Netherlands organisation for applied scientific research, where he worked first in antenna design, modeling, and simulation, and guided a few projects in the field of $\mathrm{mm}$-wave and $\mathrm{THz}$ antenna technology. In the past 7 to 8 years he worked mainly in the fields of radar signal processing and phased-array antennas, on topics like beamforming, target detection and parameter estimation, and novel waveforms. Additionally, he had the technical guidance at TNO of a few activities carried out within D-RACE, the Dutch Radar Centre of Expertise, a strategic alliance between TNO and Thales Nederland B.V. 


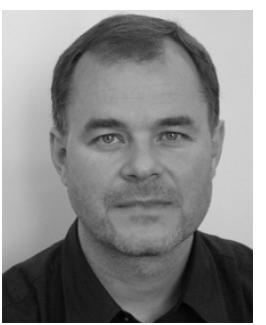

Martin N. Johansson (M'93SM'06) received the M.S. degree in Engineering Physics and the Ph.D. degree in Electromagnetics from Chalmers University of Technology, in 1986 and 1997, respectively. From 1987 to 1992, he was with Ericsson Radar Electronics AB, Mölndal, Sweden, designing antenna systems for airborne and satellite applications. During 1991, he worked for Cirrus Consulting AB on the design of the Arecibo radio telescope dualreflector feed. He joined Ericsson Research at Ericsson AB, Gothenburg, Sweden, in 1997 where he now serves as Expert in Antenna Technology. His current research interests include antenna technology for mobile communications, antenna system modeling, and deterministic propagation modeling.

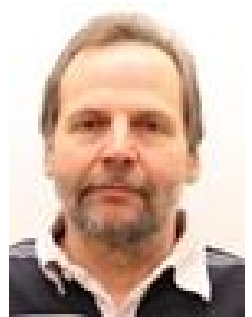

Anders Stjernman was born in 1956. He received the M.S. degree in physical engineering from Lund University in 1982 and the Ph.D. degree in space physics from Umeå University in 1995. From 1986 to 1995 , he was with the Swedish Institute of Space Physics (IRF), Kiruna, involved in radio remote sensing. From 1995 to 2002, he was with Ericsson Microwave Systems AB, where he was involved in radar antennas, radar systems, radome design, and Bluetooth antennas. Since 2002, he has been with Ericsson Research, Ericsson AB, Gothenburg, Sweden. His major areas of research are antenna theory, bandwidth limitations of antennas, and MIMO antenna system performance.

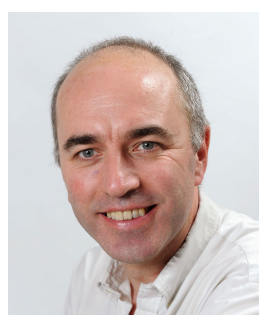

Guy A. E. Vandenbosch received the M.S. and $\mathrm{Ph} . \mathrm{D}$. degrees in Electrical Engineering from the Katholieke Universiteit Leuven, Leuven, Belgium, in 1985 and 1991, respectively. Since 1993, he has been a Lecturer, and since 2005, a Full Professor at the same university. Guy Vandenbosch has taught or teaches courses on "Electromagnetic Waves", "Antennas", "Electromagnetic Compatibility", "Fundamentals of Communication and Information Theory", "Electrical Engineering, Electronics, and Electrical Energy", and "Digital Steer- and Measuring Techniques in Physics". His research interests are in the area of electromagnetic theory, computational electromagnetics, planar antennas and circuits, nano-electromagnetics, EM radiation, EMC, and bio-electromagnetics. His work has been published in ca. 340 papers in international journals and has led to ca. 390 presentations at international conferences. In the period 19992004, he was vice-chairman, in the period 2005-2009 secretary, and in the period 2010-2016 chairman of the IEEE Benelux Chapter on Antennas and Propagation. In the period 2002-2004 he was secretary of the IEEE Benelux Chapter on EMC. In the period 2012-2014 he was secretary of the Belgian National Committee for Radio-electricity (URSI), where he is also in charge of commission E. Guy Vandenbosch is a fellow of the IEEE. From September to December 2014, he was a visiting professor at Tsinghua University, Beijing, China. Since 2017 he is a member of the IEEE Electromagnetics Award Committee.

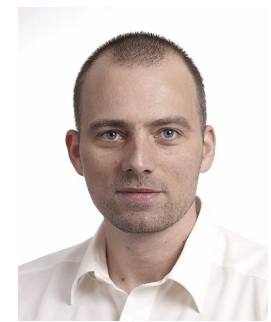

Rob Maaskant (M'11-SM'13) received the M.Sc. and $\mathrm{Ph} . \mathrm{D}$. degrees (cum laude) in electrical engineering from the Eindhoven University of Technology (TU/e), Eindhoven, The Netherlands, in 2003 and 2010, respectively. He was an Antenna Researcher at the Netherlands Institute for Radio Astronomy, Dwingeloo, The Netherlands, from 2002 to 2010. Since 2010, he has been with the Antenna Group, Signals and Systems Department, Chalmers University of Technology, Gothenburg, Sweden, where he held a postdoctoral position and was an Assistant Professor. He is currently an Associate Professor at both Chalmers University of Technology and TU/e. The latter position is owing to a five-year Vidi grant from the Dutch Research Council. He is the primary author of the CAESAR software, an advanced integralequation based solver for the analysis of large antenna array systems. His current research interest concerns the analysis and design of integrated antenna array systems for future wireless applications. Dr. Maaskant has served the AP community as an Associate Editor for the IEEE TRANSACTIONS ON ANTENNAS AND PROPAGATION and the IEEE ANTENNAS AND WIRELESS PROPAGATION LETTERS. He is on the Editorial Board of a unique open-access journal: Forum for Electromagnetic Research Methods and Application Technologies (http://www.e-fermat.org). 